\title{
El complejo amigdalino humano y su implicación en los trastornos psiquiátricos
}

\author{
The amygdaloid complex and its implication in psychiatric disorders
}

\author{
M. T. Ledo-Varela, J. M. Giménez-Amaya, A. Llamas
}

\section{RESUMEN}

El complejo amigdalino es un conjunto de núcleos que se localizan en la profundidad del lóbulo temporal, y que guardan estrecha relación con el sistema límbico. Su alteración se ha asociado a un gran número de procesos psiquiátricos. En este artículo se pretende hacer una revisión de lo publicado en referencia a la implicación amigdalina en trastornos psiquiátricos comunes. En ellos se observa un complejo amigdalino alterado, siendo su máxima expresión el síndrome de Klüver-Bucy. En pacientes esquizofrénicos se ha observado una reducción del volumen amigdalino, bilateral en varones y unilateral en mujeres. Esto sugiere que las alteraciones morfométricas del complejo amigdalino están más extendidas en varones esquizofrénicos. El complejo amigdalino está aumentado en niños autistas, no siendo así en adolescentes, donde se iguala al volumen de cualquier adolescente o adulto sano. Sin embargo, estudios neuroanatómicos han demostrado patología microscópica. En los pacientes con trastornos en el estado de ánimo, se observa una cierta tendencia a presentar un complejo amigdalino izquierdo de menor volumen. El volumen amigdalino de los grupos con demencia frontotemporal y enfermedad de Alzheimer era diferente a los del grupo de control, y se ha visto una predisposición al incremento de la atrofia amigdalina. Se puede afirmar que el complejo amigdalino está implicado en numerosos procesos psiquiátricos, tanto por daño estructural de dicho complejo como por daño funcional. Sin embargo, hacen falta más estudios para delimitar la influencia real del complejo amigdalino en dichos trastornos.

Palabras clave. Complejo amigdalino. Esquizofrenia. Autismo. Trastorno bipolar. Síndrome de Klüver-Bucy.

\begin{abstract}
The amygdaloid complex is a group of nuclei located deep in the temporal lobe and closely involved in the limbic system. Its alteration has been associated with some psychiatric processes. In this article, an overall review was made of the published data concerning the amygdaloid complex in the most common psychiatric diseases. A damaged amygdaloid complex is commonly observed, that in the Klüver-Bucy syndrome presents the fullest expression. A decrease in the amygdaloid complex of schizophrenic patients has been observed. This finding was found bilaterally in men whereas in women it was only located in one hemisphere. This finding suggests that morphometric alterations in the amygdaloid complex are more diffuse and more severe in men with schizophrenia. This subcortical complex is larger in children with autism, but not in adolescents, in whom the amygdaloid complex volume matches the normal volume of an adolescent or an adult without this pathology. However, neuroanatomical studies have shown microscopic alterations. In patients with mood disorders, it has been reported that the left amygdaloid complex presents a lesser volume. Moreover, in frontotemporal dementia and Alzheimer disease a slight amygdaloid atrophia was found related to the healthy controls. It can be concluded that the amygdaloid complex is involved in several psychiatric processes, due to structural or functional damage. However, more studies are still needed in order to delimitate the real influence of the amygdaloid complex in these disorders.
\end{abstract}

Key words. Amygdaloid complex. Schizophrenia. Autism. Bipolar disorder. Klüver-Bucy syndrome.
Departamento de Anatomía, Histología y Neurociencia. Facultad de Medicina. Universidad Autónoma de Madrid

Aceptado para su publicación el 10 de octubre de 2006

\section{Correspondencia:}

José Manuel Giménez Amaya

Departamento de Anatomía, Histología y Neurociencia

Facultad de Medicina

Universidad Autónoma de Madrid

c/ Arzobispo Morcillo, s/n

28029 Madrid

Tfno.: 914975323

Fax: 914975353

E-mail: josemanuel.gimenezamaya@uam.es 


\section{INTRODUCCIÓN}

Desde el punto de vista neuroanatómico, el complejo amigdalino sigue siendo un enigma ${ }^{1}$. Cortes sucesivos a lo largo del polo temporal de los hemisferios cerebrales nos revelan una masa gris con forma almendrada -de ahí su nombre- que ya a principios del siglo XIX Burdach descubrió y definió como complejo amigdalino ${ }^{2}$. Realmente este complejo es un conjunto de núcleos situado en la región subcortical del lóbulo temporal, en su parte anteromedial, profundo al uncus. Queda inmediatamente anterior a la formación hipocámpica y al extremo anterior del asta temporal del ventrículo lateral (Fig. 1). Es muy heterogéneo desde el punto de vista morfológico y no se considera una unidad funcional.
Está recubierto de corteza rudimentaria y, posteriormente, se continúa con el uncus del giro parahipocámpico. Las conexiones anatómicas sugieren que el complejo amigdalino es uno de los componentes clave en el circuito central de la emoción. Ciertos estudios previos han demostrado que también está implicado en aspectos del comportamiento, memoria y aprendizaje relacionados con la emoción, y más en concreto, aquellos relacionados con el miedo y la agresividad.

Con esta revisión de la literatura científica nos proponemos recopilar datos a favor de una implicación del complejo amigdalino en diferentes patologías psiquiátricas. En los apartados siguientes haremos una revisión de los trabajos publicados por
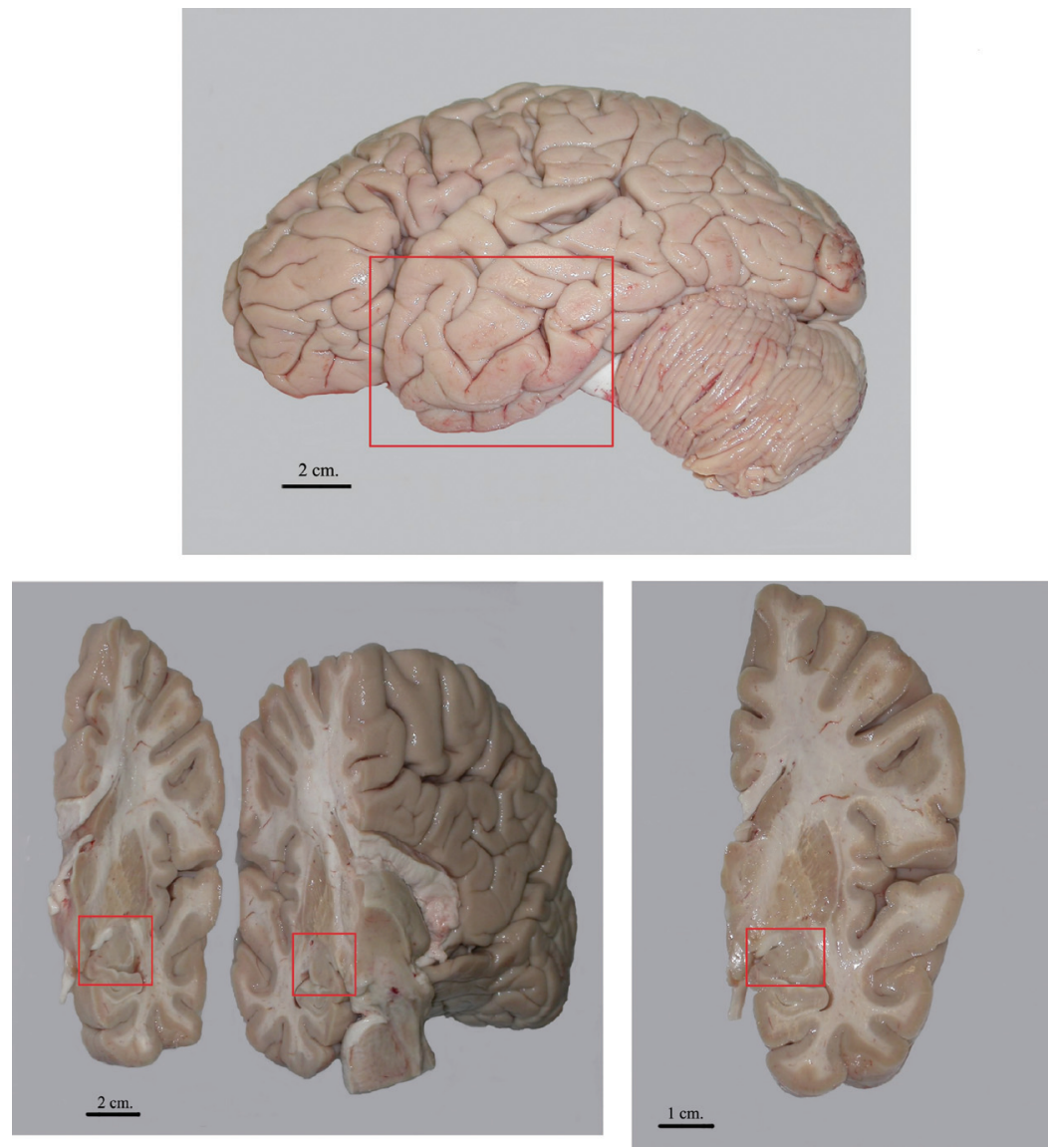

Figura 1. Localización del complejo amigdalino en el cerebro humano en fresco. 
Tabla 1. División nuclear amigdalina propuesta en nuestros estudios neuromorfológicos de esta estructura subcortical.

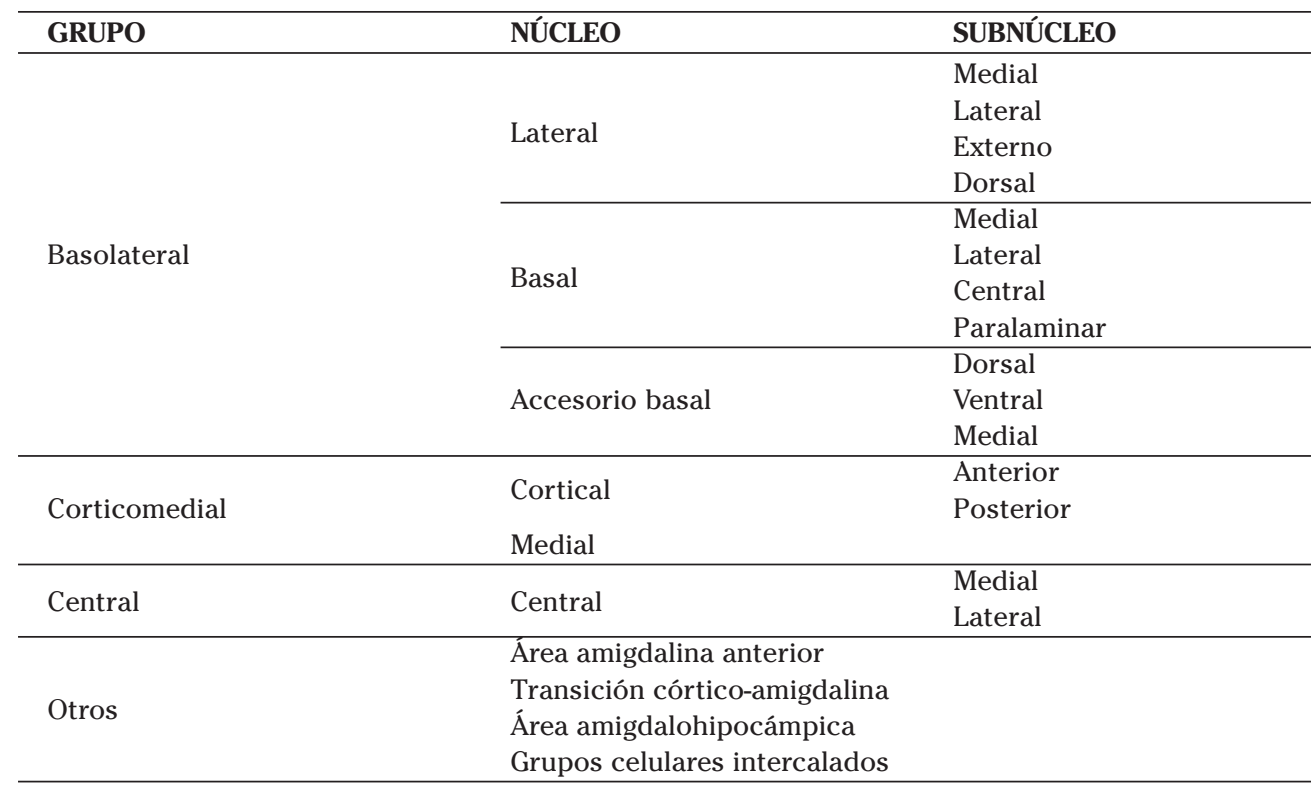

diversos autores acerca de su participación en la esquizofrenia, el autismo o los trastornos del estado de ánimo. Incluiremos finalmente también algunos comentarios sobre el síndrome de Klüver-Bucy o la enfermedad de Alzheimer por la sintomatología psiquiátrica que presentan.

\section{División nuclear}

El nombre y la clasificación de los núcleos del complejo amigdalino siguen generando controversia. Para sistematizar bien su división nuclear, hemos creído conveniente basarnos en la clasificación hecha por Sims y $\mathrm{col}^{3}$, por ser sencilla, didáctica, y muy completa y representativa de los núcleos y grupos nucleares en los que se divide este complejo, incluyendo, en los casos en los que hemos creído convenientes, ciertas modificaciones derivadas de nuestras propias observaciones (Tabla 1) (Fig. 2).

No obstante lo dicho en el párrafo anterior, existen analogías muy evidentes entre los distintos autores, de manera que se podrían establecer las correlaciones más importantes entre ellos (Tabla 2).

\section{Conexiones}

\section{a) Aferentes}

El complejo amigdalino recibe una gran cantidad de estímulos sensoriales aferentes de una forma altamente procesada ${ }^{4-6}$. Las células amigdalinas pueden responder a información aferente somatosensorial, visual, auditiva y visceral. Estas últimas, en particular las olfatorias, son especialmente importantes. Además, la información visceral llega al complejo amigdalino indirectamente desde el hipotálamo, área septal, corteza orbital e insular. La corteza temporal y la cingular anterior también proyectan al complejo amigdalino, al igual que el tronco del encéfalo, siendo la proyección más abundante la que proviene del núcleo peripeduncular. El lóbulo temporal anterior sirve de origen a la mayor parte de las fibras aferentes corticales, que se dirigen principalmente al núcleo lateral.

El complejo amigdalino ${ }^{5}$ recibe fibras aferentes serotoninérgicas desde el rafe 
Tabla 2. Correlación de las divisiones nucleares amigdalinas propuestas por distintos autores.

\begin{tabular}{|c|c|c|c|c|}
\hline $\begin{array}{l}\text { SIMS y WILLIAMS, } \\
1990\end{array}$ & BRADY, 1992 & PRICE y col 1987 & SORVARI y col 1986 & $\begin{array}{l}\text { PITKÄNEN } y \\
\text { KEMPPAINEN, } 2002\end{array}$ \\
\hline $\begin{array}{l}\text { GRUPO } \\
\text { BASOLATERAL }\end{array}$ & & & $\begin{array}{l}\text { NÚCLEOS } \\
\text { PROFUNDOS }\end{array}$ & $\begin{array}{l}\text { NÚCLEOS } \\
\text { PROFUNDOS }\end{array}$ \\
\hline $\begin{array}{l}\text { N. LATERAL } \\
\text { Medial } \\
\text { Lateral } \\
\text { Externo } \\
\text { Dorsal }\end{array}$ & N. LATERAL & $\begin{array}{l}\text { N. LATERAL } \\
\text { Dorsomedial } \\
\text { Ventrolateral }\end{array}$ & $\begin{array}{l}\text { N. LATERAL } \\
\text { Medial } \\
\text { Lateral }\end{array}$ & $\begin{array}{l}\text { N. LATERAL } \\
\text { Medial } \\
\text { Lateral }\end{array}$ \\
\hline $\begin{array}{l}\text { N. BASAL } \\
\text { Medial } \\
\text { Lateral } \\
\text { Central }\end{array}$ & $\begin{array}{l}\text { N. BASAL } \\
\text { Magnocelular } \\
\text { Parvocelular } \\
\text { Intermedio }\end{array}$ & $\begin{array}{l}\text { N. BASAL } \\
\text { Magnocelelar } \\
\text { Parvocelular }\end{array}$ & $\begin{array}{l}\text { N. BASAL } \\
\text { Magnocelular } \\
\text { Parvocelular } \\
\text { Intermedio }\end{array}$ & $\begin{array}{l}\text { N. BASAL } \\
\text { Magnocelular } \\
\text { Parvocelular } \\
\text { Intermedio }\end{array}$ \\
\hline $\begin{array}{l}\text { N. ACCESORIO } \\
\text { BASAL } \\
\text { Dorsal } \\
\text { Ventral }\end{array}$ & $\begin{array}{l}\text { N. ACCESORIO } \\
\text { BASAL } \\
\text { Magnocelular } \\
\text { Parvocelular }\end{array}$ & $\begin{array}{l}\text { N. ACCESORIO BASAL } \\
\text { Superficial } \\
\text { Magnocelular } \\
\text { Parvocelular } \\
\text { N. PARALAMINAR }\end{array}$ & $\begin{array}{l}\text { N. ACCESORIO BASAL } \\
\text { Ventromedial } \\
\text { Magnocelular } \\
\text { Parvocelular } \\
\text { N. PARALAMINAR }\end{array}$ & $\begin{array}{l}\text { N. ACCESORIO BASAL } \\
\text { Ventromedial } \\
\text { Magnocelular } \\
\text { Parvocelular } \\
\text { N. PARALAMINAR } \\
\text { Medial } \\
\text { Lateral }\end{array}$ \\
\hline $\begin{array}{l}\text { GRUPO } \\
\text { CORTICOMEDIAL }\end{array}$ & & & $\begin{array}{l}\text { NÚCLEOS } \\
\text { SUPERFICIALES }\end{array}$ & ÁREAS SUPERFICIALES \\
\hline $\begin{array}{l}\text { N. CORTICAL } \\
\text { Medial } \\
\text { Lateral } \\
\text { N. MEDIAL }\end{array}$ & $\begin{array}{l}\text { N. CORTICAL } \\
\text { Dorsal } \\
\text { Ventral } \\
\text { N. MEDIAL }\end{array}$ & $\begin{array}{l}\text { N. CORTICAL } \\
\text { Posterior } \\
\text { Anterior } \\
\text { N. MEDIAL } \\
\text { NÚCLEOS DEL TRACTO } \\
\text { OLFATORIO LATERAL }\end{array}$ & $\begin{array}{l}\text { N. CORTICAL } \\
\text { Posterior } \\
\text { Anterior } \\
\text { N. MEDIAL } \\
\text { NÚCLEOS DEL TRACTO } \\
\text { OLFATORIO LATERAL }\end{array}$ & $\begin{array}{l}\text { N. CORTICAL } \\
\text { Posterior } \\
\text { Anterior } \\
\text { N. MEDIAL } \\
\text { NÚCLEOS DEL TRACTO } \\
\text { OLFATORIO LATERAL }\end{array}$ \\
\hline GRUPO CENTRAL & & & OTROS NÚCLEOS & ÁREAS RESTANTES \\
\hline $\begin{array}{l}\text { N. CENTRAL } \\
\text { Medial } \\
\text { Lateral } \\
\text { Dorsolateral } \\
\text { Ventrolateral } \\
\text { Intersticial }\end{array}$ & $\begin{array}{l}\text { N. CENTRAL } \\
\text { Medial } \\
\text { Lateral }\end{array}$ & $\begin{array}{l}\text { N. CENTRAL } \\
\text { Medial } \\
\text { Lateral }\end{array}$ & $\begin{array}{l}\text { N. CENTRAL } \\
\text { Medial } \\
\text { Lateral }\end{array}$ & $\begin{array}{l}\text { N. CENTRAL } \\
\text { Medial } \\
\text { Lateral }\end{array}$ \\
\hline \multicolumn{5}{|c|}{ OTROS NÚCLEOS } \\
\hline $\begin{array}{l}\text { CORTEZA DE } \\
\text { TRANSICIÓN } \\
\text { AMIGDALINA } \\
\text { ÁREA } \\
\text { AMIGDALINA } \\
\text { ANTERIOR }\end{array}$ & $\begin{array}{l}\text { ÁREA DE } \\
\text { TRANSICIÓN } \\
\text { CORTICAL } \\
\text { ÁREA } \\
\text { AMIGDALINA } \\
\text { ANTERIOR }\end{array}$ & $\begin{array}{l}\text { CORTEZA } \\
\text { PERIAMIGDALINA } \\
\text { ÁREA AMIGDALINA } \\
\text { ANTERIOR }\end{array}$ & $\begin{array}{l}\text { ÁREA AMIGDALINA } \\
\text { ANTERIOR }\end{array}$ & $\begin{array}{l}\text { CORTEZA } \\
\text { PERIAMIGDALINA } \\
\text { ÁREA AMIGDALINA } \\
\text { ANTERIOR }\end{array}$ \\
\hline \multirow[t]{2}{*}{$\begin{array}{l}\text { GRUPOS } \\
\text { CELULARES } \\
\text { INTERCALADOS }\end{array}$} & $\begin{array}{l}\text { NEURONAS } \\
\text { INTERCALADAS }\end{array}$ & $\begin{array}{l}\text { NÚCLEOS } \\
\text { INTERCALADOS }\end{array}$ & $\begin{array}{l}\text { NÚCLEOS } \\
\text { INTERCALADOS }\end{array}$ & $\begin{array}{l}\text { NÚCLEOS } \\
\text { INTERCALADOS }\end{array}$ \\
\hline & & $\begin{array}{l}\text { ÁREA } \\
\text { AMIGDALOHIPOCÁMPICA } \\
\text { NÚCLEOS DEL LECHO } \\
\text { DE LA ESTRÍA TERMINAL }\end{array}$ & $\begin{array}{l}\text { ÁREA } \\
\text { AMIGDALOHIPOCÁMPI- } \\
\text { CA }\end{array}$ & $\begin{array}{l}\text { ÁREA } \\
\text { AMIGDALOHIPOCÁMPICA }\end{array}$ \\
\hline
\end{tabular}



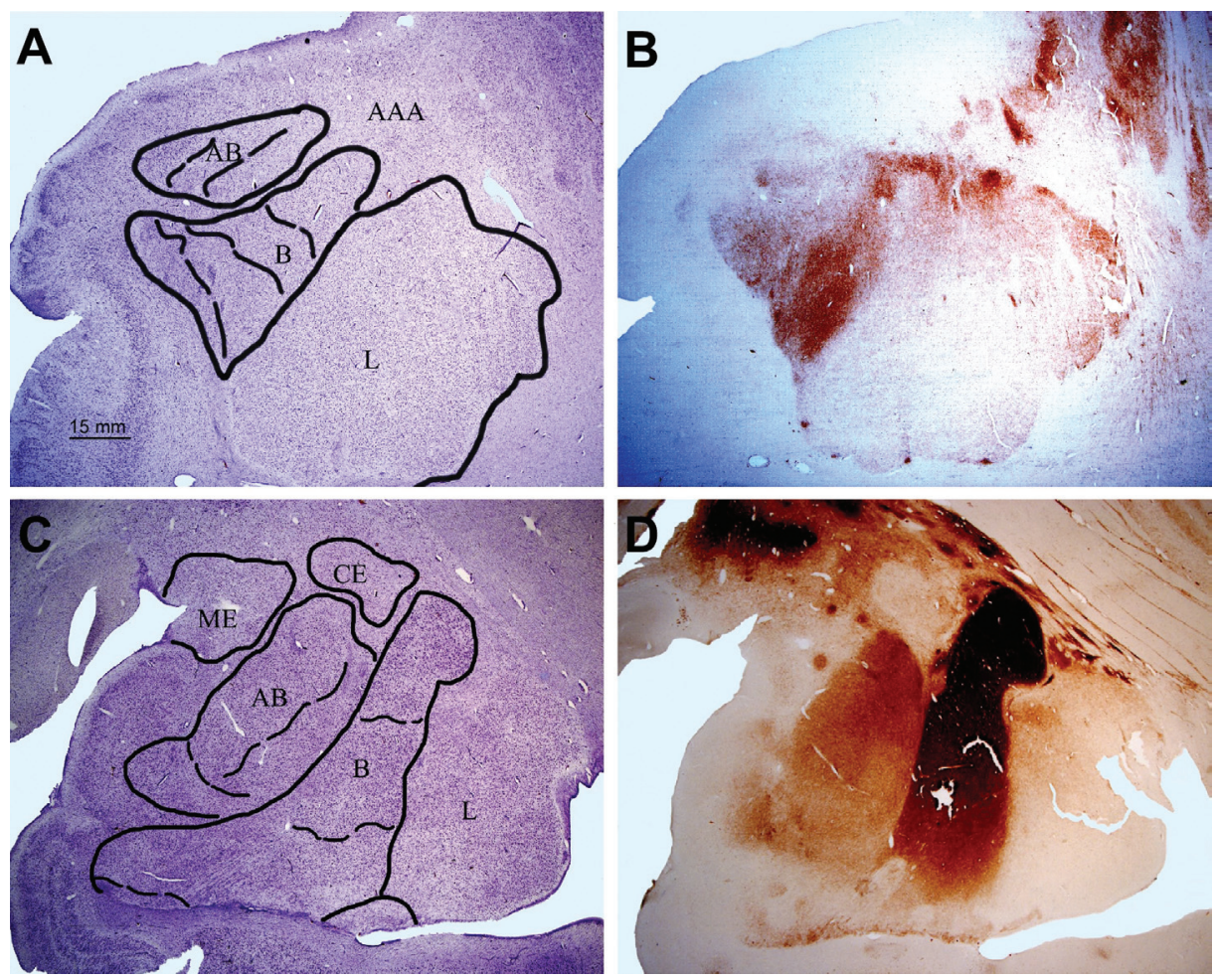

Figura 2. Cortes adyacentes de dos niveles del complejo amigdalino humano (anterior, A-B; posterior, C-D). A y C se han realizado con la técnica citoarquitectónica de Nissl, mientras que B y D se procesaron por la técnica histoquímica de la acetilcolinesterasa. Abreviaturas: AAA: área amigdalina anterior; AB: núcleo accesorio basal; B: núcleo basal; CE: núcleo central; L: núcleo lateral; ME: núcleo medial.

mesencefálico, dopaminérgicas desde el área tegmental ventral y la sustancia negra (que terminan sobre todo en el núcleo central) y noradrenérgicas desde el locus coeruleus y subcoeruleus.

Las proyecciones de retorno que van desde el hipotálamo hasta el complejo amigdalino son escasas en comparación con las proyecciones amígdalo-hipotalámicas. Proceden en gran parte del núcleo ventromedial y del área hipotalámica lateral.

Las proyecciones de retorno desde el hipocampo son menos extensas y comprenden la zona limítrofe de CA1 y el subículo adyacente, y quizás también la corteza entorrinal. Destaca la considerable polaridad de las conexiones amígdalo- hipocámpicas, ya que el complejo amigdalino parece tener mayor influencia sobre los procesos hipocámpicos que el hipocampo sobre los procesos amigdalinos.

En general, las fibras aferentes llegan al complejo amigdalino en el sentido contrario de las mismas vías seguidas por las conexiones eferentes.

\section{b) Eferentes}

Las dos vías eferentes principales del complejo amigdalino ${ }^{4}$ son la estría terminal y la vía amigdalófuga ventral.

La estría terminal es un pequeño haz de fibras que tiene por objeto llevar la información a distintas zonas encefálicas, 
entre las que cabría destacar el hipotálamo -más en concreto los núcleos preóptico, ventromedial, anterior y área hipotalámica lateral-, núcleo caudado y putamen -en especial sus áreas anteriores-, núcleo accumbens y núcleos septales, entre otros.

Tiene su origen principalmente en el grupo celular corticomedial, y de ahí discurre por el surco entre el núcleo caudado y el tálamo, acompañado en la mayor parte de su recorrido por la vena terminal, hasta llegar a sus destinos.

Cabe destacar el hecho de que en su espesor aparecen los denominados núcleos del lecho de la estría terminal, que no son más que unos acúmulos discontinuos de células que se van agregando a ella a lo largo de su recorrido.

La vía amigdalófuga ventral es el principal haz de fibras eferentes del complejo amigdalino. Este haz presenta la peculiaridad de que las distintas fibras tendrán un destino diferente según el núcleo o grupo nuclear donde se hayan originado. Así pues, los axones que provienen en su mayor parte de las células del grupo basolateral adoptarán una dirección medial a través de la sustancia innominada, para llegar finalmente al hipotálamo y núcleos septales, mientras que las fibras originadas en el núcleo central, girarán en sentido caudal, para descender de manera difusa por el tronco encefálico y terminar en los núcleos viscerales (motor dorsal del vago), núcleos del rafe (magno, oscuro y pálido) y otras áreas como el locus coeruleus, núcleos parabraquiales y sustancia gris periacueductal. La mayoría de estas áreas del tronco del encéfalo que reciben información desde el complejo amigdalino, proyectan de nuevo a esa estructura.

La sustancia innominada antes mencionada, al igual que las células del grupo basolateral, origina proyecciones de forma difusa a la corteza cerebral, en concreto a la corteza prefrontal, cingular, insular y temporal inferior. Se ha sugerido que la misión de estas fibras sería la activación de la corteza cerebral como respuesta a estímulos importantes desde el punto de vista conductual.
Cabe destacar que, si bien la mayoría de las conexiones extrínsecas del complejo amigdalino son recíprocas ${ }^{5}$, existen tres excepciones importantes: estriado, tálamo y ciertas áreas corticales, que reciben abundantes proyecciones amigdalinas, pero no las devuelven.

\section{IMPLICACIONES DEL COMPLEJO AMIGDALINO EN TRASTORNOS PSIQUIÁTRICOS}

De forma general se puede indicar que gran parte de la sintomatología neuropsiquiátrica encontrada es una manifestación común de lesiones temporolímbicas. Dichos síntomas psiquiátricos serían resultado de alteraciones de redes neuronales temporolímbicas específicas que incluyen al complejo amigdalino.

\section{Esquizofrenia}

Se sabe que el complejo amigdalino está implicado en la patología de la esquizofrenia. Los cambios estructurales y funcionales que tienen lugar en el complejo amigdalino han sido demostrados en numerosos estudios de neuroimagen ${ }^{7}$. En los últimos años se han realizado una serie de estudios morfométricos para comprobar las variaciones estructurales, en forma o tamaño, del complejo amigdalino de pacientes esquizofrénicos. La mayoría de estos estudios concluyen que hay una reducción del volumen de esta estructura subcortical en pacientes esquizofrénicos comparado con el grupo de control de sujetos sanos, aunque en ciertos estudios se hallaron resultados negativos.

Bogerts $^{8}$ realizó, en 1993, una serie de estudios neuroanatómicos con material post mortem, donde encontró lo que llamó "sutiles diferencias" con respecto a sujetos no esquizofrénicos en estructuras límbicas; entre ellas se incluía el complejo amigdalino. Las alteraciones citoarquitectónicas y la falta de gliosis en dichas estructuras límbicas, así como la ausencia de la normal asimetría estructural del cerebro en una proporción sustancial de pacientes, indican que estas anomalías estructurales pudieran reflejar una alteración prenatal en el desarrollo del cerebro. 
Niu y $\mathrm{col}^{7}$ realizaron un estudio utilizando 80 sujetos, 40 de ellos esquizofrénicos (20 hombres y 20 mujeres) de acuerdo con los criterios clínicos de diagnóstico del CIE10 , y otros 40 controles, igualmente 20 hombres y 20 mujeres. En este estudio se recogieron imágenes de resonancia magnética de todos los individuos para un posterior análisis volumétrico. Como resultado, se halló que los pacientes esquizofrénicos en su conjunto tenían una significativa reducción del volumen amigdalino en comparación con los sujetos de control, y dicha reducción fue observada bilateralmente en los varones esquizofrénicos en comparación con los individuos de control, mientras que sólo se observó reducción del volumen del complejo amigdalino derecho en las mujeres en comparación con sus correspondientes controles femeninos. Es más, se vio una significativa lateralización del volumen del complejo amigdalino en varones esquizofrénicos, de tal manera que el complejo amigdalino izquierdo era significativamente más pequeño en comparación con su correspondiente grupo de control. En resumen, en este interesante estudio se encontró una disminución del volumen amigdalino significativa en los pacientes esquizofrénicos. A esta conclusión también se había llegado previamente por otros grupos investigadores ${ }^{9,10}$.

Sin embargo, lo que resulta más interesante de este estudio es el hallazgo de una disminución bilateral del volumen amigdalino en varones pero no en mujeres, donde la reducción es sólo en el hemisferio derecho. Esto podría sugerir, como apuntan Niu y $\mathrm{col}^{7}$, que las alteraciones morfométricas del complejo amigdalino son más difusas y más prominentes en varones esquizofrénicos que en mujeres. Todo ello está en concordancia con otros estudios previos que demuestran que es más probable encontrar alteraciones morfométricas cerebrales en el varón que en la mujer, como por ejemplo el crecimiento de los ventrículos laterales ${ }^{11} \mathrm{o}$ la disminución del volumen del lóbulo temporal en su parte medial $^{12,13}$.

En cuanto al curso clínico de la enfermedad, los varones esquizofrénicos tienen mayor número de síntomas negativos, un comienzo más temprano y un peor pro- nóstico a largo plazo de la enfermedad, mientras que las mujeres tienen una mejor competencia premórbida, más signos positivos y afectivos y cuentan con una mejor respuesta al tratamiento. Geschwind y Galaburda ${ }^{14}$ postulan que el ambiente hormonal en el que crece y se desarrolla el cerebro pudiera ser también un factor fundamental a la hora de producir dimorfismos sexuales normales y, por tanto, de modular alteraciones en el cerebro en pacientes esquizofrénicos.

Sin embargo, también existen estudios, como el de Kalus y $\mathrm{col}^{15}$, en el que el examen volumétrico no evidenció alteraciones del volumen amigdalino normalizado en comparación con el grupo de control.

Finalmente señalar otros estudios en enfermos esquizofrénicos que presentan algunas particularidades. Así, Lawrie y $\mathrm{Col}^{16}$ publicaron un estudio donde queda "convincentemente demostrado" que se reduce el volumen de lo que ellos llaman complejo amígdalo-hipocámpico $(\mathrm{CAH})$ en pacientes esquizofrénicos, demostración llevada a cabo gracias a imágenes estructurales de resonancia magnética. También describen la disminución de otras zonas límbicas y paralímbicas. Pudieron comprobar igualmente que jóvenes adolescentes inicialmente sanos pero con al menos dos familiares afectos de esquizofrenia tenían un volumen de $\mathrm{CAH}$ disminuido aunque sin llegar a lo encontrado en los pacientes con esquizofrenia. Esto sugiere que posteriores reducciones de volumen pudieran estar relacionadas con la aparición de la enfermedad. Además parece ser que los volúmenes del $\mathrm{CAH}$ están genéticamente determinados en familias con un patrón de transmisión dominante.

Finalmente, Job y $\mathrm{col}^{17}$ han demostrado en concordancia con los datos hallados por Lawrie y col $^{16}$ que los pacientes con un alto riesgo de desarrollar la enfermedad tenían reducciones multifocales en la sustancia gris, y que los sujetos que habían tenido un primer episodio de esquizofrenia presentaban disminuciones multifocales en la sustancia gris en comparación con los sujetos de alto riesgo.

A la vista de lo expuesto anteriormente, se observa que, en los distintos estudios 
realizados por diferentes grupos de investigadores, se han obtenido resultados contradictorios, señalando desde que no hay ninguna diferencia significativa en los volúmenes amigdalinos de sujetos esquizofréni$\cos$ y controles ${ }^{18,19}$, hasta disminuciones notables de los volúmenes normalizados de pacientes esquizofrénicos ${ }^{20}$. Aparte de las diferencias que pudiera haber en la composición del grupo de pacientes, una de las razones de estos datos contradictorios podría ser la diferencia en los protocolos utilizados $\mathrm{y}$, en general, dificultades metodológicas a la hora de una correcta separación del complejo amigdalino respecto a estructuras hipocámpicas anteriores con imágenes de resonancia magnética.

\section{Autismo}

El autismo es un trastorno del desarrollo, que se diagnostica en la infancia ${ }^{21}$. Podría definirse también como una alteración neuropsiquiátrica que interrumpe o desbarata el desarrollo de la inteligencia social. Por inteligencia social entendemos la habilidad de interpretar la conducta de los demás en términos de estados mentales del tipo pensamientos, intenciones, deseos y creencias, y así poder relacionarse tanto en grupos sociales complejos como en relaciones cercanas $^{6}$. Otros componentes de este desorden incluyen trastornos del lenguaje, conductas estereotipadas y miedo o ansiedad desmesurada.

Desde que se describió por primera vez por Kanner ${ }^{22}$ hace ahora unos 60 años, la definición de autismo ha evolucionado mucho y en la actualidad incluye un amplio rango, basado en el rigor de las alteraciones sociales y emocionales, con diferentes niveles de función cognitiva o lingüística, pudiendo ir desde un autismo de baja funcionalidad con retraso mental, hasta otro funcionalmente alto con un coeficiente intelectual dentro de la normalidad. Casi simultáneamente a Kanner, Asperger ${ }^{23,24}$ describió un grupo de niños con un rango muy estrecho de intereses y unas relaciones sociales insuficientes, muy parecidos a los descritos como autistas de alta funcionalidad. De hecho, la distinción entre síndrome de Asperger y autismo de alta funcionalidad sigue siendo bastante controvertida.

Numerosos autores han encontrado alteraciones en el complejo amigdalino en el autismo. Hay evidencias post mortem ${ }^{25}$, que indican una densidad celular aumentada con un volumen amigdalino adulto normal. También cabe destacar la similitud que se observa entre pacientes autistas y pacientes amigdalotomizados, en los que aparece una afectación en el juicio social, que ha pasado a denominarse "autismo adquirido" ${ }^{26}$. Y un último aspecto, no menos importante, son las alteraciones estructurales y funcionales que se observan en las imágenes de resonancia magnética o de SPECT (single positron emission computed tomography), encontradas por Baron-Cohen y $\mathrm{col}^{27}$. Se sometió a un grupo de 6 sujetos con autismo de alta funcionalidad o con síndrome de Asperger, y a otros 6 individuos de control, a la realización de dos actividades. En la primera de ellas, se mostraban en un ordenador una serie de fotografías de personas, en concreto de la zona de la cara correspondiente a los ojos, y los individuos tenían que pulsar un botón de la pantalla para indicar si lo que estaban viendo era un varón o una mujer. Esta prueba se denomina "reconocimiento de género". En la segunda se pedía que se eligiera una de las dos palabras presentadas simultáneamente, la que, a juicio de cada uno de los sujetos, mejor definiera la expresión del individuo que aparecía en la fotografía (nuevamente la zona de los ojos) que se mostraba en pantalla. Estas palabras definían el posible estado de ánimo en el individuo de la fotografía. Se trataba de juzgar, por la expresión de los ojos de la otra persona presentada en la fotografía, qué era lo que dicha persona podría estar pensando o sintiendo. Es decir, se estaba poniendo a prueba la denominada "teoría de la mente", que indica, según Fine y $\mathrm{col}^{28}$, la habilidad de atribuir estados mentales a uno mismo o a otro, y de predecir y comprender el comportamiento o la conducta de otros individuos tomando como base su estado men$\mathrm{tal}^{29}$. En resumen, la diferencia clave entre estas dos tareas era el tipo de juicio a realizar por el sujeto del experimento al serle mostradas las fotografías. 
Los sujetos con autismo de alta funcionalidad o con síndrome de Asperger con un coeficiente intelectual dentro del rango considerado como normal mostraron un déficit en la realización de esta última tarea. Este grupo de pacientes activaba los componentes frontales (corteza prefrontal dorsolateral izquierda, áreas de Brodmann 44,45 y 46 y corteza frontal medial izquierda) menos extensamente que el grupo de control, pero en ningún caso se activaba el complejo amigdalino ${ }^{27}$. En cualquiera de los casos, el mayor poder de respuesta de este grupo de pacientes se observó en el giro temporal superior bilateral. Sin embargo, es interesante señalar que el grupo de control demostró un poder de respuesta significativamente mayor en el complejo amigdalino izquierdo.

Debido a las observaciones realizadas en el complejo amigdalino izquierdo, se puede suponer que esta región subcortical se ve críticamente implicada en el procesamiento de la información acerca del estado mental o emocional que proviene de estímulos visuales complejos tales como la región de los ojos. El grupo autista parece realizar la actividad sin utilizar el complejo amigdalino, y en su lugar estos pacientes parecen utilizar en gran medida las estructuras del lóbulo temporal. Esto podría entenderse como una "compensación" de dicha alteración amigdalina. En conclusión, este estudio $^{27}$ parece sugerir que la comprensión del estado mental del sujeto se procesa en el complejo amigdalino.

Por otra parte, Schumann y col$^{21}$ han demostrado que el complejo amigdalino está aumentado en niños autistas, no siendo así en adolescentes. En el desarrollo normal, el complejo amigdalino aumenta desde un tamaño de $1,7 \mathrm{~cm}^{3}$ a la edad de 8 años hasta $2,3 \mathrm{~cm}^{3}$ a la edad de 18 ; es decir, sufre un marcado aumento de volumen a una edad en la que el volumen total del cerebro varía relativamente poco. En dicho estudio se observó que los niños pequeños con autismo unido a retraso mental tenían un complejo amigdalino derecho un $16 \%$ más grande, y un complejo amigdalino izquierdo un $13 \%$ mayor que sus correspondientes controles con desarrollo normal. Niños con autismo pero sin retraso mental, también tenían un 17\% más grande tanto el complejo amigdalino derecho como el izquierdo en comparación con sus controles. En adolescentes, el volumen amigdalino se igualaba. Esto indica no sólo que el complejo amigdalino en autismo es inicialmente más grande en los niños que sus correspondientes controles con un desarrollo normal, sino que guarda una relación más estrecha con el autismo que con el retraso mental. Y por otro lado, este crecimiento amigdalino en autistas no es paralelo a un crecimiento global del cerebro, pues no hay diferencias en el volumen cerebral global del grupo de niños autistas en comparación con el grupo de control.

A la vista de todos estos datos, queda comprobada la implicación del complejo amigdalino en el autismo, tanto a edades tempranas como en la edad adulta. Sin embargo, hacen falta más estudios para saber cuál es el límite exacto de la implicación de esta estructura subcortical en dicha patología.

\section{Trastornos del estado de ánimo}

Aunque la etiología de la depresión aún no se conoce en su totalidad, los orígenes que dan lugar a este trastorno del ánimo sí son bien conocidos: biológicos, psicológi$\cos \mathrm{y}$ socioculturales $^{30}$. En los últimos 5 años, ha habido un avance sin precedentes en el conocimiento de la neurobiología de la depresión y del trastorno bipolar. Así, se han aportado nuevos datos sobre las posibles alteraciones en estas enfermedades mentales desde la genómica, la neuroimagen y la identificación de sistemas neurales implicados en la cognición, la emoción o la conducta. Además, también se han identificado nuevos marcadores, que servirán para el desarrollo de nuevos tratamientos farmacológicos, así como la identificación de numerosas variaciones genéticas asociadas a la mayoría de los desórdenes psiquiátricos ${ }^{31}$, y ya se están desarrollando numerosos test para detectar el riesgo de la enfermedad y la enfermedad propiamente dicha.

Por lo que se refiere a la depresión, se ha podido observar en estos pacientes mediante técnicas de neuroimagen que hay numerosas alteraciones a nivel de flujo 
sanguíneo en ciertas regiones cerebrales y también en cuanto al metabolismo de la glucosa -un indicador indirecto fundamental de la función neuronal- en varias regiones cerebrales, entre las que destaca el complejo amigdalino. Los estudios neuromorfológicos funcionales, sobre todo los que incluyen el complejo amigdalino, hipocampo y corteza prefrontal, han arrojado mucha luz respecto a la etiología de la depresión.

Con referencia al trastorno bipolar, Beyer y Krishnan ${ }^{32}$ señalan una amplia variedad de alteraciones neuroanatómicas en los cerebros de estos pacientes analizados con técnicas de neuroimagen. Estos hallazgos incluyen pérdida de sustancia gris, ventrículos agrandados, zonas hiperintensas vistas con resonancia magnética, así como alteraciones en el volumen regional de los ganglios basales, de estructuras laterales y mediales del lóbulo temporal (amígdala, hipocampo y tálamo) y de regiones corticales, como la corteza prefrontal dorsolateral o la corteza cingular.

Varios estudios apoyan el hecho de que hay alteraciones estructurales que afectan al complejo amigdalino en aquellos pacientes con trastorno bipolar. Strakowski y $\mathrm{Col}^{33}$ encuentran en un conjunto de pacientes tanto con primer episodio como con múltiples episodios de dicho trastorno, una diferencia global significativa en diversas estructuras en relación al grupo de control, que incluyen la corteza prefrontal, el hipocampo, el tálamo o el complejo amigdalino. De todas las estructuras afectadas, la que presentaba mayor alteración fue esta última.

Caetano y $\mathrm{col}^{34}$ realizaron un estudio donde, utilizando técnicas de resonancia magnética, midieron el volumen amigdalino y otras estructuras del lóbulo temporal en pacientes con un cuadro de depresión mayor en el momento del estudio o en remisión. Este estudio incluyó a pacientes con depresión mayor no medicados y controles sanos. Se encontró una cierta disminución en el volumen del complejo amigdalino izquierdo en el conjunto de pacientes con depresión en comparación con los controles. Estos hallazgos están en concordancia con otros trabajos previos que llegaron a la misma conclusión ${ }^{35}$.

La mayoría de las alteraciones que se mencionan en todos los estudios ${ }^{36}$ se refieren a circuitos límbico-tálamo-corticales y límbico-estriado-pálido-talámicos, estructuras que han sido implicadas en la fisiopatología de los trastornos del ánimo.

Por último, señalar que los cambios en el volumen amigdalino podrían representar un marcador biológico primario, o bien una adaptación a episodios repetidos de dicha enfermedad. Un estudio realizado por Altshuler y $\mathrm{col}^{37}$ apoya esta teoría a favor de una relación entre episodios maníacos previos y un volumen amigdalino alterado, aunque aún existe cierta controversia sobre este tema.

\section{Otros trastornos neuropsiquiátricos asociados}

\section{Síndrome de Klüver-Bucy}

El síndrome de Klüver-Bucy clásico o trastorno bilateral del lóbulo temporal se considera una consecuencia directa de la destrucción bilateral del complejo amigdalino $^{38}$, donde frecuentemente se ven también afectadas estructuras vecinas como la corteza temporal inferior y la sustancia blanca que las rodea; en conjunto esta destrucción da como resultado un síndrome neuro-conductual típico. La descripción inicial tras experimentos con monos incluía las siguientes características: hiperoralidad (tendencia a examinar todos los objetos con la boca); comportamiento dietético indiscriminado (con aceptación de muchos tipos de comida anteriormente rechazados); hipermetamorfosis (tendencia a atender y a reaccionar frente a cualquier estímulo visual); actividad autoerótica enormemente aumentada (homo y heterosexual, con elección inapropiada del sujeto); mansedumbre y falta de temor; agnosia visual y capacidad de manipular toda clase de objetos sin distinguir entre peligrosos y no peligrosos ${ }^{39}$. La implicación del complejo amigdalino en este síndrome se ha demostrado en numerosos estudios. Uno de los más conocidos es el de Horel y col ${ }^{40}$, que publicaron que habían conseguido 
reproducirlo en el laboratorio, observando los síntomas de dicho síndrome en aquellos monos donde se había producido una destrucción de esta estructura subcortical.

La incidencia en humanos del síndrome suele ocurrir típicamente como resultado de lesiones quirúrgicas, meningoencefalitis $^{41}$, o enfermedades catalogadas como raras, por ejemplo, la enfermedad de Pick. Aparece asociado también a una variedad de desórdenes neurológicos, como en la encefalopatía anóxico-isquémica, encefalitis por herpes simple, neurocisticercosis, traumatismo craneoencefálico con gliosis o meningitis tuberculosa ${ }^{42}$.

En el ser humano, la lesión bilateral de los lóbulos temporales, que afecta también al uncus e hipocampo en la mayoría de los casos, origina el mismo síndrome que aquel que se obtiene en laboratorio con monos. Los síntomas típicos en el hombre incluyen: bulimia o hiperfagia, es decir, comer objetos que no son apropiados; hiperoralidad o tendencia en exceso a explorar y a contactar oralmente con objetos incomibles o a olerlos (incluso pueden examinar un objeto dañino, como una cerilla encendida, llevándosela a los labios o tocándola con la lengua) y un impulso irrefrenable de tocar objetos. También se pierde la capacidad de reconocer objetos con la vista (agnosia visual) y puede existir asociada una agnosia táctil y auditiva. No es infrecuente observar en el paciente la compulsión de explorar a fondo el ambiente cercano (hipermetamorfosis) y reaccionar exageradamente a los estímulos visuales.

Es llamativa la pérdida del sentimiento de miedo, enojo o ira ${ }^{4}$, incluso cuando dicha reacción sea la oportuna, observándose en el paciente una placidez característica. Aparece de igual modo una hipersexualidad o aumento llamativo de la conducta sexual, que se puede poner en evidencia en la forma de conversaciones impúdicas y vagos intentos de contacto sexual. Además de estos problemas previsibles, los pacientes también pueden experimentar amnesia, demencia o afasia, dependiendo de la extensión de la lesión en el lóbulo temporal.

\section{Demencia y enfermedad de Alzheimer}

La demencia frontotemporal (DFT) es una enfermedad neurodegenerativa caracterizada por una amplia serie de alteraciones en el comportamiento. Algunas de estas alteraciones incluyen la desinhibición, la hiperoralidad y la hipersexualidad, rasgos éstos que recuerdan al síndrome de Klüver-Bucy. Esto podría apuntar a la implicación del complejo amigdalino en la aparición de dichos síntomas, como sugieren Boccardi y $\mathrm{col}^{43}$. De hecho, ciertos estudios, como el de Jack y col ${ }^{44}$, demuestran que el complejo amigdalino de estos pacientes con DFT es atrófico comparado con los sujetos de control.

En un estudio llevado a cabo por Boccardi y $\mathrm{col}^{43}$ se refirió una pérdida de alrededor del $20 \%$ en el complejo amigdalino de pacientes con DFT. Y lo que es más, la atrofia amigdalina tendía a aumentar en pacientes con enfermedad de Alzheimer (EA), hasta llegar a un 30\%. Según Jack y $\mathrm{Col}^{44}$, el volumen amigdalino, observado por medio de técnicas de neuroimagen, es significativamente menor en pacientes con EA que en sujetos de control. Sin embargo, el hecho de que la atrofia del complejo amigdalino sea menor en la DFT que en la EA, parecería no tener correspondencia con la observación clínica que indica que los síntomas que presumiblemente guardan mayor relación con un daño amigdalino son más evidentes en la DFT que en la EA. Una posible explicación a esto sería que esos síntomas no se deben a una alteración amigdalina per se, sino al trastorno de un sistema neural, que comprendería a esta estructura subcortical y que incluiría a los lóbulos frontales, especialmente dañados en la DFT, y en estrecha relación con el complejo amigdalino. Esta hipótesis también está de acuerdo con las suposiciones de otros autores, como Carroll y $\mathrm{col}^{45,46}$, los cuales hallaron síntomas del tipo Klüver-Bucy en pacientes sin una alteración amigdalina clara, y lo atribuyeron entonces a una alteración de las conexiones fronto-límbicas.

Finalmente, añadir que en un reciente estudio neuropatológico de las alteraciones de la anatomía química del complejo amigdalino en la EA han demostrado que 
existe un déficit colinérgico en el núcleo basal lateral de estos pacientes ${ }^{47}$.

\section{CONCLUSIONES}

Se ha comprobado en repetidos estudios que las diversas alteraciones en la estructura del complejo amigdalino están implicadas en la patogenia de diferentes enfermedades mentales. Así pues, vemos cómo una disminución en el volumen de dicho complejo se relaciona con enfermedades de tipo esquizofrenia. Se observa una disminución de volumen de manera bilateral en los varones afectos de esquizofrenia, en quienes la sintomatología de esta enfermedad es, en líneas generales, más cruda, y su respuesta al tratamiento es, en conjunto, menor. En mujeres esquizofrénicas también se ha observado, aunque de manera unilateral, dicha disminución de volumen amigdalino. De igual modo, se han constatado otras alteraciones estructurales, como crecimiento de los ventrículos laterales y disminución del volumen del lóbulo temporal en su parte medial. Otro trastorno con cambio estructural amigdalino claro es el de la alteración del ánimo, donde se ha encontrado un volumen amigdalino izquierdo disminuido.

Hay otros procesos, en cambio, que aunque guardan poca relación con alteraciones estructurales amigdalinas -encontramos un complejo amigdalino dentro de límites anatómicos considerados como normales-, tienen una función de dicha estructura subcortical ampliamente alterada. Tal es el caso del autismo. En experimentos realizados con sujetos adultos que padecían autismo de alta funcionalidad o con sujetos con síndrome de Asperger -ambas entidades de las que es difícil establecer un diagnóstico diferencial-, se observó que el volumen amigdalino era similar en ambos grupos, no pudiendo encontrarse diferencias macroscópicas. Sin embargo, cuando se requería a dichos sujetos a realizar una determinada tarea, en concreto, la de atribuir estados mentales a otros sujetos viendo sólo sus ojos, los sujetos de control activaban de manera importante su complejo amigdalino, especialmente el izquierdo, mientras que los pacientes con las citadas patologías activaban otras estructuras telencefálicas, pero en ningún caso activaban el complejo amigdalino. En su lugar, como una "compensación", el mayor poder de respuesta se observó en el giro temporal superior bilateral. Se veía así claramente la implicación del complejo amigdalino en la patogenia de ciertos aspectos del autismo, en concreto en la habilidad de la que tan característicamente carecen los sujetos con autismo, que es la denominada "inteligencia social". No se han podido demostrar alteraciones en el volumen amigdalino en adultos con autismo, aunque sí en niños. En estos últimos se ha comprobado que existe un volumen amigdalino aumentado. Pero parece suceder que al llegar a la adolescencia, se iguala el tamaño de esta estructura subcortical.

Pero no sólo las alteraciones estructurales o funcionales del complejo amigdalino están implicadas en las distintas patologías, sino que la destrucción bilateral del complejo amigdalino, o bien la destrucción de las vías que lo conectan con el resto del encéfalo, puede originar por sí sola una enfermedad: el síndrome de Klüver-Bucy. En ella, el paciente pierde la capacidad de reconocer los objetos que percibe $o$, siendo más precisos, pierde la capacidad de dotar de cualidades emocionales al objeto que tiene delante. Es incapaz de distinguir si ese objeto es apto para ser ingerido, por ejemplo. Esto se traduce en la hiperoralidad, o la tendencia que tienen estos pacientes a oler el objeto, ya que necesitan valerse de otros medios para saber de qué objeto se trata. En este ejemplo se aprecia la gran implicación del complejo amigdalino en dotar de cualidades afectivas a lo material, a lo externo, incluyendo por supuesto al miedo o la ansiedad ante diversas situaciones vitales, todo ello características principales atribuibles al complejo amigdalino.

A la vista de todo lo expuesto anteriormente, queda clara la gran implicación del complejo amigdalino en numerosas patologías psiquiátricas o trastornos neuropsiquiátricos relacionados. Faltan aún numerosos estudios por realizar, para confirmar o reforzar los hallazgos obtenidos hasta el momento, pero, a la luz de los datos actua- 
les, el complejo amigdalino se revela como una estructura importante para descifrar el enigma de estas enfermedades mentales que son todavía hoy tan difíciles de comprender desde el punto de vista neurobiológico.

\section{BIBLIOGRAFÍA}

1. Amaral DG, Price JL, Pitkänen A, CARmichael ST. Anatomical organization of the primate amygdaloid complex. En: Appelton JP, editor. The amygdala: neurobiological aspects of emotion, memory and mental dysfunction. New York: Wiley-Liss, Inc, 1992: 1-66.

2. Swanson LW, Petrovich GD. What is the amygdala? Trends Neurosci 1998; 21: 323331.

3. Sims KS, WiLLIAMS RS. The human amygdaloid complex: a cytologic and histochemical atlas using Nissl, myelin, acetylcholinesterase and nicotinamide adenine dinucleotide phosphate diaphorase staining. Neuroscience 1990; 36: 449-472.

4. HAINES DH. Principios de Neurociencia. $2^{\mathrm{a}}$ ed. Madrid: Elsevier, 2003.

5. AmARAL DG. Amygdalohippocampal and amygdalocortical projections in the primate brain. Adv Exp Med Biol 1986; 203: 3-17.

6. BARon-Cohen S, Ring HA, Bullmore ET, WheElright S, Ashwin C, Williams SCR. The amygdala theory of autism. Neurosci Behav Rev 2000; 24: 355-364.

7. Niu L, Matsui M, Zhou S, Hagino H, TAKahashi T, YONEYAMA E et al. Volume reduction of the amygdala in patients with schizophrenia: a magnetic resonance imaging study. Psychiatry Res: Neuroimaging 2004; 132: 4151.

8. Bogerts B. Recent advances in the neuropathology of schizophrenia. Schizophr Bull 1993; 19: 431-445.

9. Breier A, Buchanan RW, Elkashef A, Munson RC, KirkPATRICK B, Gellard F. Brain morphology and schizophrenia. A magnetic resonance imaging study of limbic, prefrontal cortex and caudate structures. Arch Gen Psychiatry 1992; 49: 921-926.

10. Pearlson GD, Barta PE, Powers RE, Menon RR, RichaRdS SS, AYLWARd EH et al. Medial and superior temporal gyral volumes and cerebral asymmetry in schizophrenia versus bipolar disorder. Biol Psychiatry 1997; 41: 114.

11. ANdREASEN NC, EhrhardT JC, SWAYze VW, Alliger RJ, Yuh WT, CoHEN G. Magnetic resonance imaging of the brain in schizophrenia. The pathophysiologic significance of structural abnormalities. Arch Gen Psychiatry 1990; 47: 35-44.

12. Bogerts B, Ashtari M, Degreef G, Alvir JM, BILDER RM, LiEBERMAN JA. Reduced temporal limbic structure volumes on magnetic resonance images in first episode of schizophrenia. Psychiatry Res: Neuroimaging 1990; 35: 1-13.

13. Shenton ME, Dickey CC, Frumin M, McCarley RW. A review of MRI findings in schizophrenia. Schizophr Res 2001; 49: 1-52.

14. Geschwind N, Galaburda AM. Cerebral lateralization. Biological mechanisms, associations, and pathology: II. A hypothesis and a program for research. Arch Neurol 1985; 42: 521-552.

15. Kalus P, Slotboom J, Gallinat J, Wiest R, Ozdoba C, Federspiel A et al. The amygdala in schizophrenia: a trimodal magnetic resonance imaging study. Neurosci Lett 2005; 375: 151-156.

16. LAWRIE SM, Whalley HC, Job DE, Johnstone EC. Structural and functional abnormalities of the amygdala in schizophrenia. Ann N Y Acad Sci 2003; 985: 445-460.

17. Job DE, Whalley HC, McConnell S, Glabus M, JOHnSTONE EC, LAWRIE SM. Voxel-based morphometry of grey matter densities in subjects at high risk of schizophrenia. Schizophr Res 2003; 64: 1-13.

18. Altshuler LL, Bartzokis G, Grieder T, Curran J, JIMENEZ T, LEIGHT $\mathrm{K}$ et al. An MRI study of temporal lobe structures in men with bipolar disorder or schizophrenia. Biol Psychiatry 2000; 48: 147-162.

19. Niemann K, Hammers A, Coenen VA, Thron A, KLOSTERKOTTER J. Evidence of a smaller left hippocampus and left temporal horn in both patients with first episode schizophrenia and normal control subjects. Psychiatry Res: Neuroimaging 2000; 99: 93-110.

20. Joyal CC, LaAkso MP, Tilhonen J, Syvalahti E, VILKMAN H, LAAKSO A et al. The amygdala and schizophrenia: a volumetric magnetic resonance imaging study in first-episode, neuroleptic-naïve patients. Biol Psychiatry 2003; 54: 1302-1304.

21. Schumann CM, Hamstra J, Goodlin-Jones BL, LOTSPeich LJ, KwON H, BuONOCORE MH et al. The amygdala is enlarged in children but not adolescents with autism; the hippocampus is enlarged at all ages. J Neurosci 2004; 24 : 6392-6401.

22. KANNER L. Autistic disturbances of affective contact. Nerv Chil 1943; 2: 217-250. 
23. AsPerger H. Die autistischen Psychopathen im Kindersalter. Arch Psychiat Nervenkrank 1944; 177: 76-137.

24. Hippler K, KLICPERA C. A retrospective analysis of the clinical case records of "autistic psychopaths" diagnosed by Hans Asperger and his team at the University Children`s Hospital, Vienna. Philos Trans R Soc Lond B Biol Sci 2003; 358: 291-301.

25. RAPIN I, KATZMAN R. Neurobiology of autism. Ann Neurol 1998; 43: 7-14.

26. Adolphs R, Tranel D, Damasio H, Damasio A. Impaired recognition of emotion in facial expressions following bilateral damage to the human amygdala. Nature 1994; 372: 669672 .

27. BARON-CoHen S, Ring HA, WheElWright S, Bullmore ET, BrAmmer MJ, Simmons A et al. Social intelligence in the normal and autistic brain: an fMRI study. Eur J Neurosci 1999; 11: 1891-1898.

28. Fine C, Lumsden J, Blair RJR. Dissociation between "theory of mind" and executive functions in patients with early left amygdala damage. Brain 2001; 124: 287-298.

29. Premack D, Woodruff G. Does the chimpanzee have a theory of mind? Behav Brain Sci 1978; 1: 515-526.

30. Kalia M. Neurological basis of depression: an update. Metabolism 2005; 54: 24-27.

31. Caspi A, Sugden K, Moffit Te, Taylor A, Craig IW, HARRINGTON $\mathrm{H}$ et al. Influences of life stress on depression: moderation by a polymorphism in the 5-HTT gene. Science 2003; 301: 386-389.

32. BEYER JL, KRISHNAN KR. Volumetric brain imaging findings in mood disorders. Bipolar Disord 2002; 4: 89-104.

33. Strakowski SM, DelBello MP, Sax KW, Zimmerman ME, SHEAR PK, HaWkins JM et al. Brain magnetic resonance imaging of structural abnormalities in bipolar disorder. Arch Gen Psychiatry 1999; 56: 254-260.

34. Caetano SC, Hatch JP, Brambilla P, Sassi RB, Nicoletti M, Mallinger AG et al. Anatomical MRI study of hippocampus and amygdala in patients with current and remitted major depression. Psychiatry Res: Neuroimaging 2004; 132: 141-147.

35. Von Gunther A, Fox NC, Cipolotti L, Ron MA. A volumetric study of hippocampus and amygdala in depressed patients with subjective memory problems. J Neuropsychiatry Clin Neurosci 2000; 12: 493498.

36. HajeK T, CARREy N, Alda M. Neuroanatomical abnormalities as risk factors for bipolar disorders. Bipolar Disord 2005; 7: 393-403.

37. Altshuler Ll, Bartzokis G, Grieder T, Curran J, Jimenez T, LEIGHT K et al. An MRI study of temporal lobe structures in men with bipolar disorder or schizophrenia. Biol Psychiatry 2000; 48: 147-162.

38. Hayman LA, Rexer JL, Pavol MA, Strite D, MEYERS CA. Klüver-Bucy syndrome after bilateral selective damage of amygdala and its cortical connections. J Neuropsychiatry Clin Neurosci 1998; 10: 354-358.

39. Kolb B, Whishaw IQ. Fundamentos de Neuropsicología Humana. Barcelona: Labor, 1986.

40. Horel JA, Keating EG, Misantone LJ. Partial Klüver-Bucy syndrome produced by destroying temporal neocortex or amygdala. Brain Res 1975; 94: 347-359.

41. Marlowe WB, Mancall BL, Thomas JJ. Complete Klüver-Bucy syndrome in man. Cortex 1975; 11: 53-59.

42. JHA S, PATEL R. Klüver-Bucy syndrome. An experience with six cases. Neurol India 2004; 52: 369-371.

43. Boccardi M, Pennanen C, LaAkso MP, Testa C, Geroldi C, Soininen $\mathrm{H}$ et al. Amygdaloid atrophy in frontotemporal dementia and Alzheimer's disease. Neurosci Lett 2002; 335: 139-143.

44. JaCk CR Jr, Petersen RC, Xu YC, Waring SC, O’Brien PV, TANGalos EG et al. Medial temporal atrophy on MRI in normal aging and very mild Alzheimer's disease. Neurology 1997; 49: 786-794.

45. Carroll BT, Goforth HW, Raimonde LA. Partial Klüver-Bucy syndrome: two cases. CSN Spectrums 2001; 6: 329-332.

46. Carroll BT, Goforth HW, Carroll LA. Anatomic basis of Klüver-Bucy syndrome. J Neuropsychiatry Clin Neurosci 1999; 11: 116.

47. Sahin HA, Emre M, Ziabreva I, Perry E, Calasum $\mathrm{B}$, PERRY R. The distribution pattern of pathology and cholinergic deficits in amygdaloid complex in Alzheimer's disease and dementia with Lewy bodies. Acta Neuropathol 2006; 111: 115-125. 